\title{
Anion recognition and induced self-assembly of an $\alpha, \gamma$-cyclic peptide to form spherical clusters
}

\author{
Nuria Rodríguez-Vázquez, ${ }^{[a]}$ Manuel Amorín, ${ }^{[a]}$ Ignacio Alfonso, ${ }^{[b]}$ Juan R. Granja*[a]
}

Dedication ((optional))

\begin{abstract}
A cyclic octapeptide composed of hydroxyl-functionalized $\gamma$-amino acids folds in a ' $v$-shaped' conformation that allows the selective recognition of anions such as chloride, nitrate and carbonate. The process involves the simultaneous self-assembly of six peptide subunits and the recognition of four anions to form a tetrahedral structure in which the anions are located at the corners of the resulting structure. Each anion is coordinated to three different peptides. The structure was fully characterized by several techniques, including NMR spectroscopy and X-ray diffraction, and the material was able to facilitate the transmembrane transport of chloride.
\end{abstract}

The ability to construct complex functional materials using a small number of components represents an economical synthetic strategy that allows the rapid generation of novel functions ${ }^{[1,2]}$ Anion recognition is quite a difficult task due to their larger size compared with isoelectronic cations that, as a consequence, establish weaker interactions with the receptor. ${ }^{[3]}$ In addition, anions are more susceptible to $\mathrm{pH}$ changes losing their charge at low $\mathrm{pH}$ values. Furthermore, anions have a wide range of geometries and high free energies of hydration. All of these limitations demand a high level of design and synthesis to produce effective recognition receptors and this has retarded the development of such systems. Nevertheless, in the last few years this topic has emerged as a fundamental area in chemistry with applications in a variety of fields, such as catalysis, ion extraction and in responsive chemical systems. ${ }^{[4]}$ Anions have an enormous impact upon our lives due to the fundamental roles that they play in biological events. ${ }^{[5]}$ Apart from organic anions (ATP, RNA or DNA), inorganic anions, such as chloride, carbonate or phosphate, are found extensively in extracellular fluids and their dysregulation is associated with diseases such as cystic fibrosis and bone mineralization. ${ }^{[6]}$ The processes outlined above, amongst others, make it of fundamental importance to understand the nature of the anion recognition process to improved receptor designs.

[a] Ms. N. Rodríguez-Vázquez, Dr. M. Amorín, Prof. Dr. J. R. Granja Departamento de Química Orgánica and Centro Singular de Investigación en Química Biológica y Materiales Moleculares (CIQUS)

Universidad de Santiago de Compostela

15782 Santiago de Compostela (Spain)

E-mail: juanr.granja@usc.es

[b] Dr. I. Alfonso

Departamento de Química Biológica y Modelización Molecular Instituto de Química Avanzada de Cataluña (IQAC-CSIC) Jordi Girona, 18-26

08034 Barcelona (Spain)

Supporting information for this article is given via a link at the end of the document. CCDC 1443051 contains the supplementary crystallographic data for this paper. These data are provided free of charge by the Cambridge Crystallographic Data Centre.
In recent years new designs have been developed in which molecular recognition is combined with a self-assembly process to form supramolecular gels, crystals or metalorganic cages. ${ }^{[7]}$ Despite these recent advances, more efficient designs are required in which small molecules merge symbiotically with specific guests (anion) to form discrete and well-defined supramolecular entities with novel properties. The use of biologically compatible systems, such as peptides, that can be involved in relevant bioprocesses is an area of great interest. ${ }^{[8]}$ The study described here concerns a new and efficient combination of a supramolecular self-assembly process of a cyclic peptide templated by specific anion recognition to form discrete spherical aggregates in which four anions in a tetrahedral disposition are coordinated to six cyclic peptides.

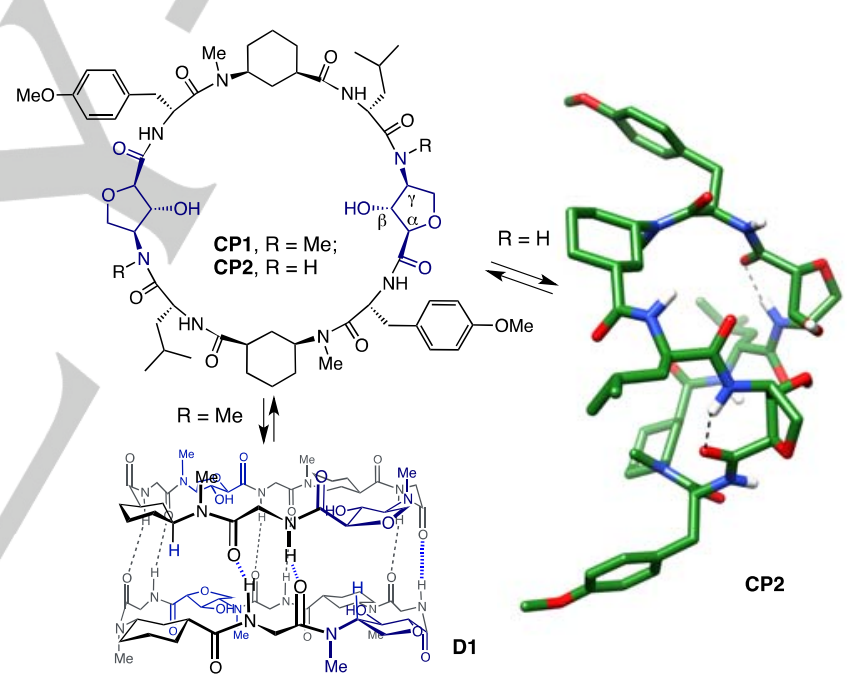

Scheme 1. Structure of cyclic peptides CP1 and CP2 and the expected equilibrium with the corresponding dimers (D1 and D2), side chains were removed for clarity. On the right, the DFT calculated structure of CP2 using experimental structural restrictions.

In recent years we have been working with cyclic peptides (CPs) composed of $\gamma$-amino acids in the search for peptide nanotubes. ${ }^{[9,10]}$ With the aim of tuning the internal properties of the nanotube (Scheme 1), ${ }^{[11]}$ 4-amino-3-hydroxytetrahydrofuran1-carboxylic acid (Ahf) was prepared. ${ }^{[12]}$ The incorporation of two $\mathrm{N}$-methylated Ahf residues in a cyclic octapeptide together with two $N$-methylated $\gamma$-amino acids [3-aminocyclohexanecarboxylic acid (Ach)] (CP1, R = Me) gave the corresponding dimer D1 (Scheme 1). ${ }^{[11 \mathrm{~b}]}$ In contrast, the CP with the non-methylated Ahf residues $(\mathbf{C P 2}, \mathrm{R}=\mathrm{H})$ did not form the corresponding dimer (D2). The characteristic nature of the NMR spectrum (Figure 1SI) in non-polar solvents led us to study their properties. In the ${ }^{1} \mathrm{H}$ NMR spectrum a downfield shift was observed for the three amide protons (9.27, 8.62 and $8.24 \mathrm{ppm})$. The chemical shift of 
the Ahf amide proton (8.62 ppm) and the singlet signals for the two vicinal protons at $\mathrm{C} 2$ and $\mathrm{C} 3$ suggest that the peptide adopts a folded conformation in which the $\mathrm{NH}$ is hydrogen bonded to its own carbonyl group. ${ }^{[13]}$ The DFT calculations, with experimental constrictions such as the aforementioned coupling constants between $\mathrm{H} \alpha, \mathrm{H} \beta$ and $\mathrm{H} \gamma$ and the participation of $\mathrm{N}-\mathrm{H}$ (Ahf) in a hydrogen bond, suggested that CP2 adopts the folded conformation illustrated in Scheme $1 .^{[14]}$ Variable concentration experiments were carried out and only a small upfield shift was

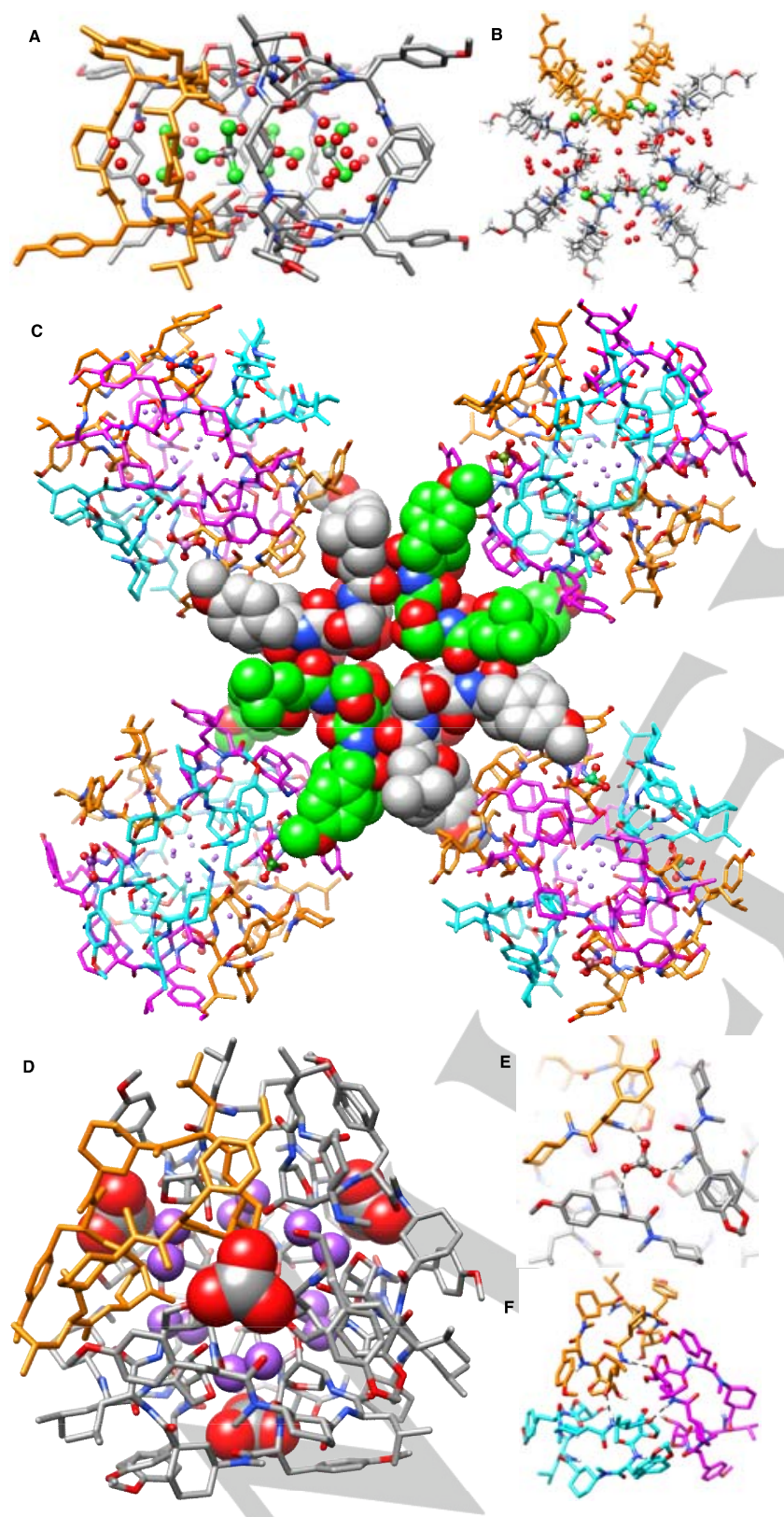

Figure 1. Crystal structure of CP2; side (A) and top (B) view of the tetrameric structure; $(C)$ view of the packing of hexamers and tetramers, (D) view of the hexameric structure in which each carbonate is coordinated to three different CPs, such interaction is based on three hydrogen bonds (E) between amide protons of Tyr and carbonate anion. (F) Hydrogen bond interaction between Leu residues stabilizing the hexameric structure. observed for the Ahf $\mathrm{NH}$ (from 8.62 to $8.55 \mathrm{ppm}$ from 20 to 2.5 $\mathrm{mM}$, Figure 2SI). The addition of small amounts of methanol (1$5 \%)$ produced a small downfield shift in the resonances of the amide protons. The addition of larger amounts of methanol $(>10 \%)$ led to the appearance of new signals that correspond to other conformations. These structures were the only ones remaining after 48 hours under these conditions (Figure $3 \mathrm{SI}$ ). Unfortunately, the symmetry of CP2 did not allow to determine the solution structure due to the lack of clear nOe cross peaks.

The nature of the structure was tried to clarify by carrying out a crystallization study. It was found that small crystals were formed from a chloroform solution of CP2 in a hexane-saturated atmosphere. Two different supramolecular units were found (Figure 1). One of the aggregates is a tetrameric structure in which the CP is folded in a 'V-shaped' conformation similar to that predicted by DFT calculations with the aforementioned Ahf hydrogen bond restriction (Scheme 1). The tetramer is constituted by two independent molecules, one of which is conformationally disordered (Figure $1 \mathrm{~B}$ and Figure 4SI). The main conformation, with a refined occupancy of $68 \%$, is almost coincident with the other independent molecule of the tetramer (green color in Figure 4SI). The $\alpha$-amino acids participate in hydrogen bonds with the neighboring CPs through a $\beta$-sheettype interaction with Leu residues hydrogen bonded to Tyr (Figure 5SI).

Each of these peptides interacts through its concave face with the second supramolecular structure (Figure $1 \mathrm{C}$ ). This cluster is a hexameric aggregate formed by three independent molecules (Figure 6SI). One of the CPs shows conformational disorder at the $\operatorname{Tyr}(\mathrm{Me})$ side chain. To our surprise, this hexamer contain four carbonate ions that establish interactions (hydrogen bonded to the Tyr amide proton) with three different CPs (Figure 1D and 1E). As carbonate was not added, it was inferred that CP2 must scavenge this anion from the drying of the chloroform solution carried out for crystallization. The resulting tetrahedral structure has the carbonate located in each corner. The $\mathrm{NH}$ of Leu is hydrogen bonded to the carbonyl of Leu of the closed $\mathrm{CP}$, which also has a trimeric organization (Figure $1 \mathrm{~F}$ ). This structure that is electrically neutral contains eight sodium ions occupying sixteen positions ( 0.5 occupancy each). The resulting 'V-shaped' structure is more compact than those that form the tetrameric aggregate or the DFT-calculated structure (Figure 7SI and 8SI). Intrigued by the formation of this anion cluster we decided to investigate the anion (carbonate) recognition in solution. Thus, NMR studies were carried out with the addition of organic solvent-soluble salts. Treatment of a $\mathrm{CDCl}_{3}$ solution of CP2 (5 $\mathrm{mM}$ ) with small portions of bistetrabutylammonium carbonate (BTBAC, Figure 2) led to downfield shifts for some of the signals in the ${ }^{1} \mathrm{H}$ NMR spectrum of CP2. Of particular significance was the change observed for the Tyr NH (9.27 to $9.59 \mathrm{ppm}$ ), which is responsible for the carbonate interaction in the crystal structure. The other $\mathrm{NHs}$ are also shifted downfield, albeit to a lesser extent (Leu NH from $8.25 \mathrm{ppm}$ to $8.32 \mathrm{ppm}$ and 8.62 to 8.68 for the Ahf $\mathrm{NH}$ ). The singlet for the proton at $\mathrm{C} \beta$ also changed from $4.21 \mathrm{ppm}$ to a broad singlet at $4.29 \mathrm{ppm}$, suggesting some conformational changes at this position. Only $\sim 0.6$ equivalents of BTBAC were required to achieve the maximum downfield shift and this corresponds to the stoichiometry observed in the solid state. Further addition of carbonate did not change the spectrum. 

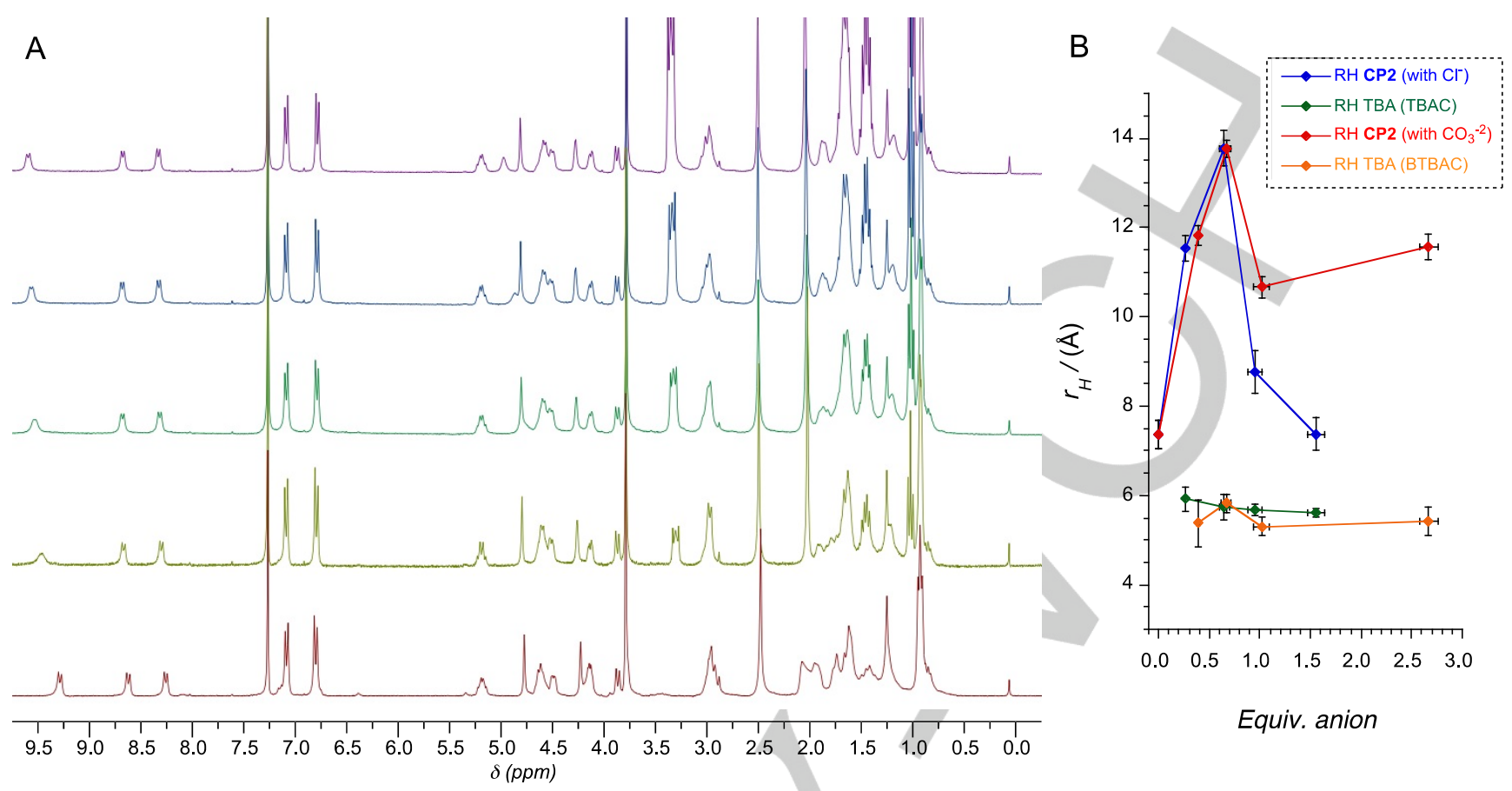

Figure 2. (A) ${ }^{1} \mathrm{H}$ NMR spectra for the addition of $\mathrm{BTBAC}(0.0,0.2,0.4,0.6$ and 1.0 equiv, bottom to top) to a CDCl 3 solution of $\mathrm{CP2}$ (5 mM). (B) Plot of the hydrodynamic radii $\left(r_{\mathrm{H}}, \AA\right)$ measured by DOSY $\left(500 \mathrm{MHz}, \mathrm{CDCl}_{3}, 25^{\circ} \mathrm{C}\right)$ for samples containing $\mathrm{CP} 2(2.55 \mathrm{mM})$ and different proportions of $\mathrm{BTBAC}$ and TBAC. The values for CP2 are shown in red (BTBAC) or blue (TBAC), while the values for the TBA cation are represented in orange (BTBAC) and green (TBAC).

The addition of tetrabutylammonium bicarbonate ${ }^{[15]}$ (TBABC, Figure 9SI) gave very different results. Initially, the addition of small amounts of bicarbonate produced the downfield shift of the $\mathrm{NH}$ signals that was followed by signals splitting, especially those corresponding to amide protons. Further addition of TBABC led to a reduction in the intensity of the amide proton signals while the intensity of the chloroform signal increased. These changes must correspond to a proton-deuterium exchange between the amide and chloroform catalyzed by the bicarbonate. In any case, definitive evidence for the formation of any supramolecule was not found. Similar results to BTBAC were obtained on addition of chloride (TBAC, Figure 10SI) or hydroxide (TBAH, Figure 11SI). Their ${ }^{1} \mathrm{H}$ NMR spectra showed changes in the chemical shifts of the amide protons, thus confirming their participation in hydrogen bonding interactions with the amide proton of Tyr. Titration with nitrate (TBAN, Figure 12SI) also produced the downfield shift of the amide protons, albeit to a lesser extent and without saturation, even in the presence of 4 equivalents of anion, suggesting a weaker binding interaction. The addition of softer anions, such as iodide (TBAI, Figure 13SI), barely modified the peptide spectrum and this suggests weaker recognition of these ions.

Considering the ${ }^{1} \mathrm{H}$ NMR titration results obtained with carbonate and chloride anions, we attempted to gain further insights into the supramolecular structures formed in solution. To this end, Diffusion Ordered Spectroscopy (DOSY) was carried out on samples containing CP2 (2.55 mM in $\mathrm{CDCl}_{3}$ ) and increasing proportions of the target anions (as the TBA salts). The selfdiffusion rates obtained in DOSY experiments were compared with those measured for tetrakis(trimethylsilyl)silane (TMSS).
The results were used to estimate the apparent hydrodynamic radii $\left(r_{\mathrm{H}}\right)$ of the species formed in solution (see supporting information for details). The apparent $r_{\mathrm{H}}$ in the absence of anions was measured to be $7.4 \pm 0.3 \AA$, which is compatible with the size of CP2 (Figure 2B). Interestingly, the size of the molecule increased during the titration experiment, with a maximum value at ca. 0.67 equivalents of anion. This value corresponds to a 3:2 CP2:anion ratio and was obtained for both carbonate and chloride ions (red and blue symbols, respectively, in Figure 2B). The maximum $r_{\mathrm{H}}$ value obtained with 0.67 equivalents of anion was $\sim 13.8 \pm 0.4 \AA$, which is in good agreement with the size of the hexameric assembly observed in the solid state, with a calculated radius of gyration of $\sim 13.9-14.2 \AA$. The addition of an excess of anion led to a decrease in the apparent size of the CP in solution. These observations suggest that the hexameric structure was formed in solution in the presence of the appropriate amount of anion, but a large excess produce species with larger CP:anion ratios. In any case, the clear maximum observed (Figure $2 \mathrm{~B}$ ) imply a high stability for the anion-templated assembly of CP2. The apparent molecular size of the TBA cation was also monitored during the titration and this remained virtually constant at $r_{\mathrm{H}} \sim 5.4-5.9 \AA$, which is consistent with its dimensions, and inferring that it is not interacting with the hexameric aggregate.

The transport of anions across phospholipid bilayers is crucial to maintain the concentration gradients used for signaling and cellular regulation. ${ }^{[16]}$ The ability of $\mathbf{C P 2}$ to transport anions across phospholipid bilayers was assessed using fluorescence techniques. These experiments were carried out in large unilamellar vesicles (200 nm diameter) and the anion-selective 
probe lucigenin was used (Figure 3). ${ }^{[17]}$ The vesicles enclosed $\mathrm{NaNO}_{3}$ with the halide-sensitive dye lucigenin $(1.0 \mathrm{mM})$ were suspended in a isosmotic solution containing $\mathrm{NaCl}(24 \mathrm{mM})$. After 70 seconds CP2 was added and the decay in lucigenin fluorescence upon the influx of chloride was measured over time. As expected, the transport rate increased upon addition of CP2. At $\mathrm{CP}$ concentrations above $20 \mu \mathrm{M}$ the transport experiments were quite erratic and the formation of a white precipitate was observed. Unfortunately, this low solubility did not allow to carry out a Hill analysis. ${ }^{[18]}$ Control studies, monitoring the release of internal dye (carboxyfluorescein) indicated that the changes in fluorescence intensity was not due to vesicles rupture (liposome leakage) (Figure 14SI). ${ }^{[19]}$ The anion transport (Figure 3) was expected to be antiporter, with chloride exchanged by intravesicular nitrate. In an effort to confirm this hypothesis, the nitrate was replaced by hydrophilic sulfate. Under the latter conditions the rate of fluorescence decay was negligible compared to the background. Therefore the inward anion $\left(\mathrm{Cl}^{-}\right)$ flow cannot be balanced under these conditions. This result confirms that the transport is an antiporter process in which the CP2 can transport chloride or nitrate but is unable to transport sulfate.
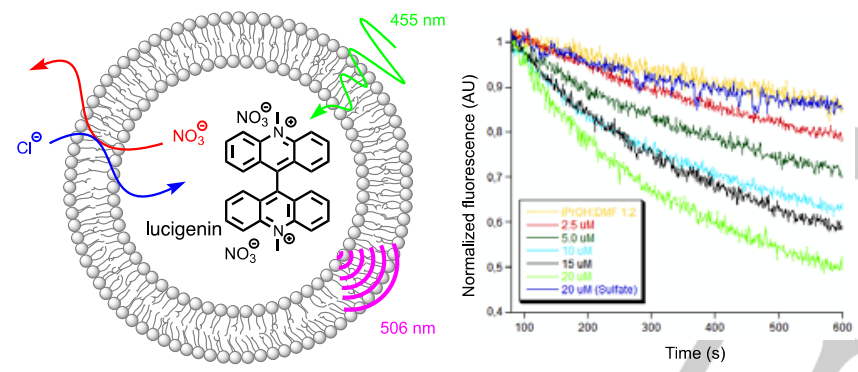

Figure 3. Transport activities were measured in synthetic vesicles using the lucigenin assay, in which fluorescence quenching due to incoming chloride was detected. On the right, representative normalized fluorescence kinetics of transport-mediated reduction of fluorescence, following addition of CP2 with variable concentrations between 2.5 and $20 \mu \mathrm{M}$ in cuvette $(0.6-5 \mathrm{~mol} \% \mathrm{CP}$ to lipid ratio).

In summary, a new supramolecular-based anion recognition process is reported in which self-assembly and molecular recognition combine to form a hexameric cluster. The process is based on a cyclic peptide (CP2) containing two 4-amino-3hydroxytetrahydrofuran-1-carboxylic acid residues that induce the peptide to fold in a ' $v$-shaped' conformation. This conformation recognizes biologically relevant anions that induce self-assembly to form a supermolecule composed of six CPs and four anions. The data clearly demonstrate that CP2 has an extremely high affinity for carbonate and other anions, such as chloride, nitrate or hydroxide. The high anion affinity and the hydrophobicity of the resulting cluster enable efficient anion transport through biological membranes. The peptidic character of this cluster might allow its implementation in relevant processes of biological interest. Efforts in this direction will be reported in due course.

\section{Acknowledgements}

This work was supported by the Spanish Ministry of Economy and Competitivity (MINECO) and the ERDF [CTQ2013-43264-R, CTQ2012-38543-C03-03], and by the Xunta de Galicia and the ERDF (GPC2013-039 and EM2014/011). N. R.-V. thanks MINECO for her FPU contract. We also thank ORFEO-CINCA network and MINECO (CTQ2014-51912-REDC). Technical support from the Unidade de Raios X, RIADT, USC, and especially to Antonio L. Llamas-Saiz is gratefully acknowledged.

Keywords: Cyclic peptide $\bullet$ self-assembly • anion recognition • supramolecular chemistry $\bullet$ transport

[1] A. C. Mendes, E. T. Baran, R. L. Reis, H. S. Azevedo, WIREs Nanomed. Nanobiotechnol. 2013, 5, 582-612.

[2] J. W. Steed, Nat. Chem. 2011, 3, 9-10.

[3] P. A. Gale, Chem. Commun. 2010, 47, 82-86; N. Busschaert, C Caltagirone, W. Van Rossom, P. A. Gale, Chem. Rev. 2015, 115, 8038-8155; S. Kubik, Chem. Soc. Rev. 2010, 39, 3648-3663.

[4] N. H. Evans, P. D. Beer, Angew. Chem. Int. Ed. 2014, 53, 11716-11754; Angew. Chem. 2014, 1216, 11908-11948.

[5] F. M. Ashcroft, Ion Channels and Disease, Academic Press, San Diego, USA, 2000.

[6] S. M. Rowe, S. Miller, E. J. Sorscher, N. Engl. J. Med. 2005, 352, 19922001; P. A. Gale, N. Busschaert, C. J. E. Haynes, L. E. Karagiannidis, I. L. Kirby, Chem. Soc. Rev. 2014, 43, 205-241.

[7] R. Custelcean, Chem. Soc. Rev., 2010, 39, 3675-3684; M.-O. M. Piepenbrock, G. O. Lloyd, N. Clarke, J. W. Steed, Chem. Rev. 2010, 110, 1960-2004; I. A. Riddell, M. M. J. Smulders, J. K. Clegg, Y. R. Hristova, B. Breiner, J. D. Thoburn, J. R. Nitschke, Nat. Chem. 2012, 4, 751-756.

[8] R. B. P. Elmes, K. A. Jolliffe, Chem. Commun. 2015, 51, 4951-4968; S. Kubik, R. Goddard, R. Kirchner, D. Nolting, J. Seidel, Angew. Chem. Int. Ed. 2001, 40, 2648-2651; Angew. Chem. 2001, 113, 2722-2725.

[9] M. Amorín, L. Castedo, J. R. Granja, J. Am. Chem. Soc. 2003, 125, 2844-2845; M. Amorín, R. J. Brea, L. Castedo, J. R. Granja, Org. Lett. 2005, 7, 4681-4684; R. J. Brea, M. Amorín, L. Castedo, J. R. Granja, Angew. Chem. Int. Ed. 2005, 44, 5710-5713; Angew. Chem. 2005, 117, 5856-5859.

[10] R. Garcia-Fandino, M. Amorín, L. Castedo, J. R. Granja, Chem. Sci. 2012, 3, 3280-3285; J. Montenegro, C. Vázquez-Vázquez, A. Kalinin, K. E. Geckeler, J. R. Granja, J. Am. Chem. Soc. 2014, 136, 2484-2491; M. Cuerva R. García-Fandiño, C. Vázquez-Vázquez, M. A. López-Quintela, J. Montenegro, J. R. Granja, ACS Nano 2015, 9, 10834-10843.

[11] a) C. Reiriz, M. Amorín, R. García-Fandiño, L. Castedo, J. R. Granja, Org. Biomol. Chem. 2009, 7, 4358-4361; b) N. Rodríguez-Vázquez, R. GarcíaFandiño, M. Amorín, J. R. Granja, Chem. Sci. 2016, 7, 183-187.

[12] N. Rodríguez-Vázquez, S. Salzinger, L. F. Silva, M. Amorín, J. R. Granja, Eur. J. Org. Chem. 2013, 3477-3482.

[13] A. A. Edwards, G. J. Sanjayan, S. Hachisu, G. E. Tranter, G. W. J. Fleet, Tetrahedron Lett. 2006, 62, 7718-7725.

[14] R. Garcia-Fandino, L. Castedo, J. R. Granja, S. Vázquez J. Phys. Chem B 2010, 114, 4973-4983; W. J. Hehre, L. Radom, P. v. R. Schleyer, J. A. Pople, Ab Initio Molecular Orbital Theory, 1st ed.; Wiley: New York, 1986.

[15]C. T. Yang, Y. Fu, Y. B. Huang, J. Yi, Q. X. Guo, L. Liu, Angew. Chem. Int. Ed. 2009, 48, 7398-7401; Angew. Chem. 2009, 121, 7534-7537.

[16] N. Busschaert, P. A. Gale, Angew. Chem. Int. Ed. 2013, 52, 1374-1382; Angew. Chem. 2013, 125, 1414-1422; J. T. Davis, O. Okunola, R. Quesada Chem. Soc. Rev. 2010, 39, 3843-3862.

[17] B. A. McNally, A. V. Koulov, B. D. Smith, J. B. Joos, A. P. Davis, Chem. Commun. 2005, 1087-1089; M. Lisbjerg, H. Valkenier, B. M. Jessen, H. AlKerdi, A. P. Davis, M. Pittelkow, J. Am. Chem. Soc. 2015, 137, 4948-4951.

[18]T. Takeuchi, N. Sakai, S. Matile, Faraday Discuss. 2009, 143, 187-203. 
Entry for the Table of Contents (Please choose one layout)

Layout 1:

\section{COMMUNICATION}

Text for Table of Contents

Layout 2:

\section{COMMUNICATION}
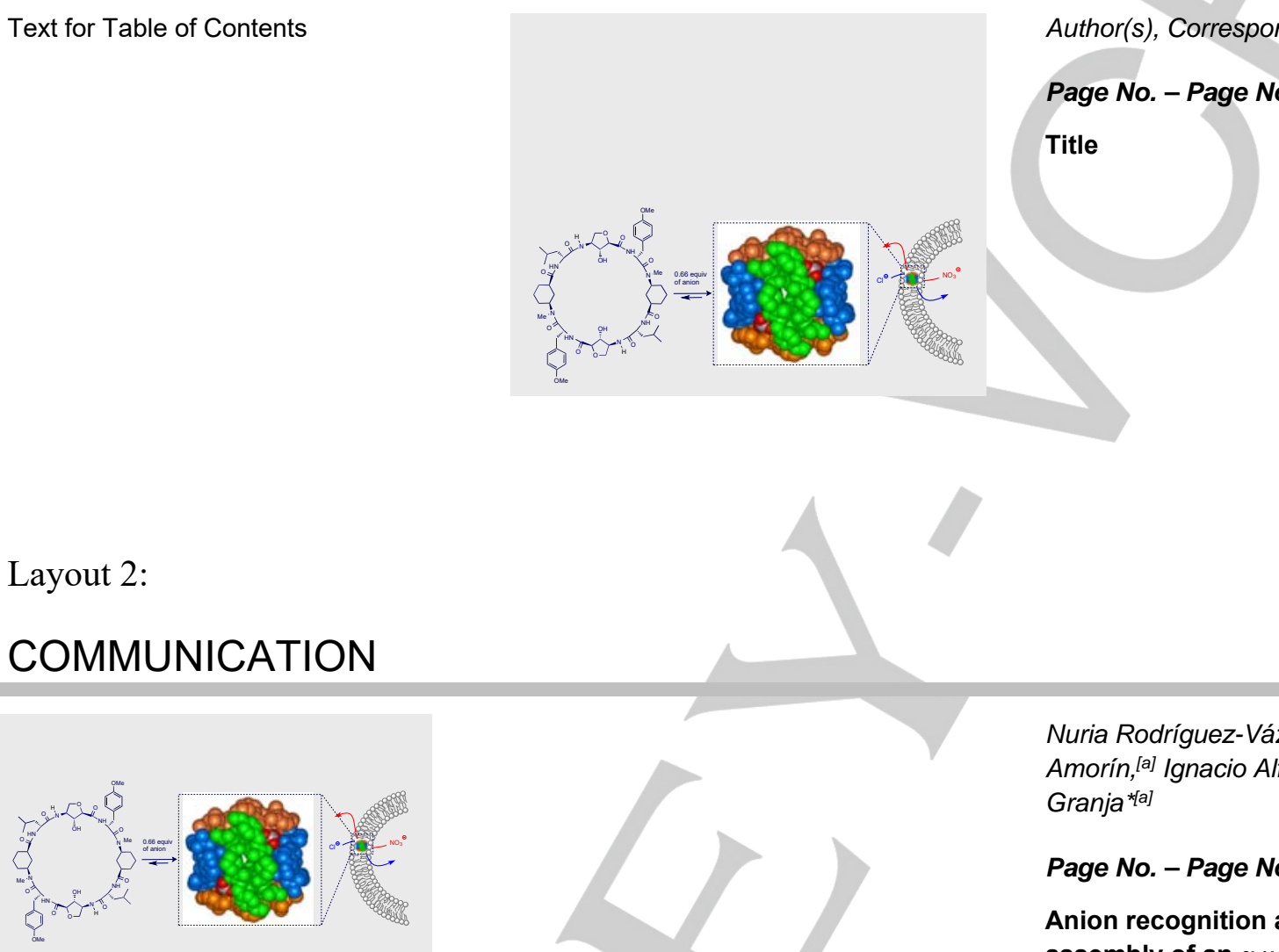

A selective anion recognition process is described that involves the simultaneous assembly of six cyclic peptide subunits and four anions to give a supramolecular cluster that efficiently facilitates transmembrane transport of the anions.
Nuria Rodríguez-Vázquez, ${ }^{[a]}$ Manuel Amorín, ${ }^{[a]}$ Ignacio Alfonso, ${ }^{[b]}$ Juan $R$. Granja*[a] $^{*}$

Page No. - Page No.

Anion recognition and induced selfassembly of an $\alpha, \gamma$-cyclic peptide to form spherical clusters 